\title{
Changes in public order after the opening of a medically supervised safer injecting facility for illicit injection drug users
}

\author{
Evan Wood, Thomas Kerr, Will Small, Kathy Li, David C. Marsh, Julio S.G. Montaner, \\ Mark W. Tyndall
}

Abstract

Background: North America's first medically supervised safer injecting facility for illicit injection drug users was opened in Vancouver on Sept. 22, 2003. Although similar facilities exist in a number of European cities and in Sydney, Australia, no standardized evaluations of their impact have been presented in the scientific literature.

Methods: Using a standardized prospective data collection protocol, we measured injection-related public order problems during the 6 weeks before and the 12 weeks after the opening of the safer injecting facility in Vancouver. We measured changes in the number of drug users injecting in public, publicly discarded syringes and injection-related litter. We used Poisson log-linear regression models to evaluate changes in these public order indicators while considering potential confounding variables such as police presence and rainfall.

Results: In stratified linear regression models, the 12-week period after the facility's opening was independently associated with reductions in the number of drug users injecting in public $(p<$ $0.001)$, publicly discarded syringes $(p<0.001)$ and injectionrelated litter $(p<0.001)$. The predicted mean daily number of drug users injecting in public was 4.3 (95\% confidence interval $[\mathrm{Cl}] 3.5-5.4)$ during the period before the facility's opening and 2.4 (95\% Cl 1.9-3.0) after the opening; the corresponding predicted mean daily numbers of publicly discarded syringes were 11.5 (95\% Cl 10.0-13.2) and 5.4 (95\% Cl 4.7-6.2). Externally compiled statistics from the city of Vancouver on the number of syringes discarded in outdoor safe disposal boxes were consistent with our findings.

Interpretation: The opening of the safer injecting facility was independently associated with improvements in several measures of public order, including reduced public injection drug use and public syringe disposal.

CMAJ 2004;171(7):731-4

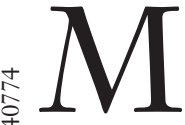

any cities are experiencing epidemics of bloodborne diseases as a result of illicit injection drug use, ${ }^{1-3}$ and drug overdoses have become a leading cause of death in many urban areas. ${ }^{4-6}$ Public drug use also plagues many inner city neighbourhoods, and the unsafe disposal of syringes in these settings is a major community concern. ${ }^{7-13}$

In over 2 dozen European cities and, more recently, in Sydney, Australia, medically supervised safer injecting facil- ities, where injection drug users (IDUs) can inject previously obtained illicit drugs under the supervision of medical staff, have been established in an effort to reduce the community and public health impacts of illicit drug use. ${ }^{14}$ Inside these facilities IDUs are typically provided with sterile injecting equipment, emergency care in the event of overdose, as well as primary care services and referral to addiction treatment. ${ }^{13,15}$ Although anecdotal reports have suggested that such sites may improve public order, ${ }^{12}$ reduce the number of deaths from overdose ${ }^{16}$ and improve access to care ${ }^{17}$ no standardized evaluations of their impact are available in the scientific literature. ${ }^{18}$

On Sept. 22, 2003, health officials in Vancouver opened a government-sanctioned safer injecting facility as pilot project. The facility, the first in North America, is centrally located in Vancouver's Downtown Eastside, which is the most impoverished urban neighbourhood in Canada and home to well-documented overdose and HIV epidemics among the estimated 5000 IDUs who reside there. ${ }^{19,20}$ Federal approval for the 3-year project was granted on the condition that the health and social impacts of the facility be rigorously evaluated. Although evaluation of the facility's impact on certain outcomes (e.g., HIV incidence) is ongoing and will take several years, it is now possible to examine the impacts of the site on public order. Therefore, we conducted this study to test the hypothesis that changes in improperly discarded syringes and public drug use would be observed after the opening of the safer injecting facility.

\section{Methods}

The present study was designed before the opening of the safer injecting facility in Vancouver's Downtown Eastside and involved standardized data collection protocols that were developed before the surveyor was trained and before the study protocol was implemented in the field. The city of Vancouver's activities for collecting used syringes were not modified during the study period, to avoid this potential source of confounding. The study design was approved by the University of British Columbia / Providence Healthcare Research Ethics Board more than 3 months before the opening of the safer injecting facility.

The survey protocol involved measuring specified public order indicators within a predefined geographic area and at predefined times of the week during the 6 weeks before and the 12 weeks after 
the facility opened. Specifically, we obtained maps of the neighbourhood's network of roads and alleyways and selected a predefined study area consisting of the 10 city blocks that surrounded the safer injecting facility. Data collection times were spread evenly throughout the week and involved walking through the study zone in the same pattern from 10 am to noon on Monday, from $1 \mathrm{pm}$ to $3 \mathrm{pm}$ on Wednesday and from $3 \mathrm{pm}$ to $5 \mathrm{pm}$ on Friday each week. One of us (W.S.), who had over 3 years' experience conducting ethnographic research in the neighbourhood and is trained in environmental surveying and mapping techniques, conducted all of the field surveys.

We identified 5 indicators of public disorder for measurement. Public injection drug use, publicly discarded syringes and injectionrelated litter were identified as measures of public drug use. Injectionrelated litter was defined as syringe wrappers, syringe caps, sterile water containers and "cookers" (containers used to heat drugs before injection). We chose to measure injection-related litter in addition to discarded syringes because Vancouver has multiple locations for syringe distribution and return. The city's largest exchange location has observed a return rate of used syringes of about $95 \%,{ }^{21}$ and although publicly discarded syringes are not an uncommon sight, syringerelated litter is a much more prevalent sign of public drug use in the neighbourhood, because wrappers and other debris are not often returned to needle exchange sites..$^{22,23}$ For the fourth indicator of public disorder, we counted the number of suspected drug dealers as a background measure, since we assumed that this variable would not be directly affected by the facility's opening. Finally, because law enforcement activities are known to have an impact on the location of injection drug use,,$^{21,24}$ we also evaluated the total number of police patrols that were encountered during the hours of data collection.

Measurements were taken for 6 weeks before and 12 weeks after the opening of the safer injecting facility. We chose these 2 periods to obtain sufficient follow-up to afford statistical power while minimizing the potential effect of seasonal changes on drug use patterns. In addition, because we recognized that rainfall patterns could still confound rates of public drug use and other public order measures, we also obtained daily rainfall statistics from Environment Canada for the days measurements were taken. ${ }^{2}$

We applied a statistical protocol, defined a priori, to examine the potential relation between the public order measures and the operation of the safer injecting facility. First, for the presentation of the crude weekly data, we recognized that measures within the same week would likely be highly correlated. ${ }^{8}$ Therefore, for each public order measure, we calculated a daily average for each week from the 3 daily counts that week. To test for changes in the various measures, we compared the daily averages for the 6 -week period before the opening of the facility with the daily averages for the 12-week period after the opening, using the Wilcoxon rank-sum test for nonnormally distributed data. Second, we recognized that, if there were a relation between the public order measures and the operation of the facility, it would likely be highly dependent on the rate of use of the facility. We therefore evaluated the number of times that the facility was used by IDUs on the days data were collected and tested for correlations between daily use of the facility and the daily counts of each public order measure using Spearman's correlation coefficient. Third, we fit Poisson log-linear regression models with the daily counts of each of the public order measures as the dependent variable and potential explanatory variables (e.g., police presence, rainfall) as the independent variables. Although most IDUs do not discard their syringes in public in Vancouver, each public order measure was considered in separate regression models because we assumed that the measures would be highly correlated. ${ }^{21}$ We examined the indepen- dent variables in unadjusted linear regression models and then adjusted for rainfall, police presence and study period (before v. after the facility's opening). Parameter estimates from the unadjusted regression models were used to calculate the predicted mean daily numbers (and 95\% confidence intervals [CIs]) of IDUs injecting in public, publicly discarded syringes and injection-related litter in the 2 study periods. Finally, as an external measure of the impact of the safer injecting facility on public drug use, we examined data from the city of Vancouver on the number of syringes discarded in the 6 outdoor safe disposal boxes in the study area during the 2 study periods. All $p$ values were 2 -sided, with a significance level of $p<0.05$.

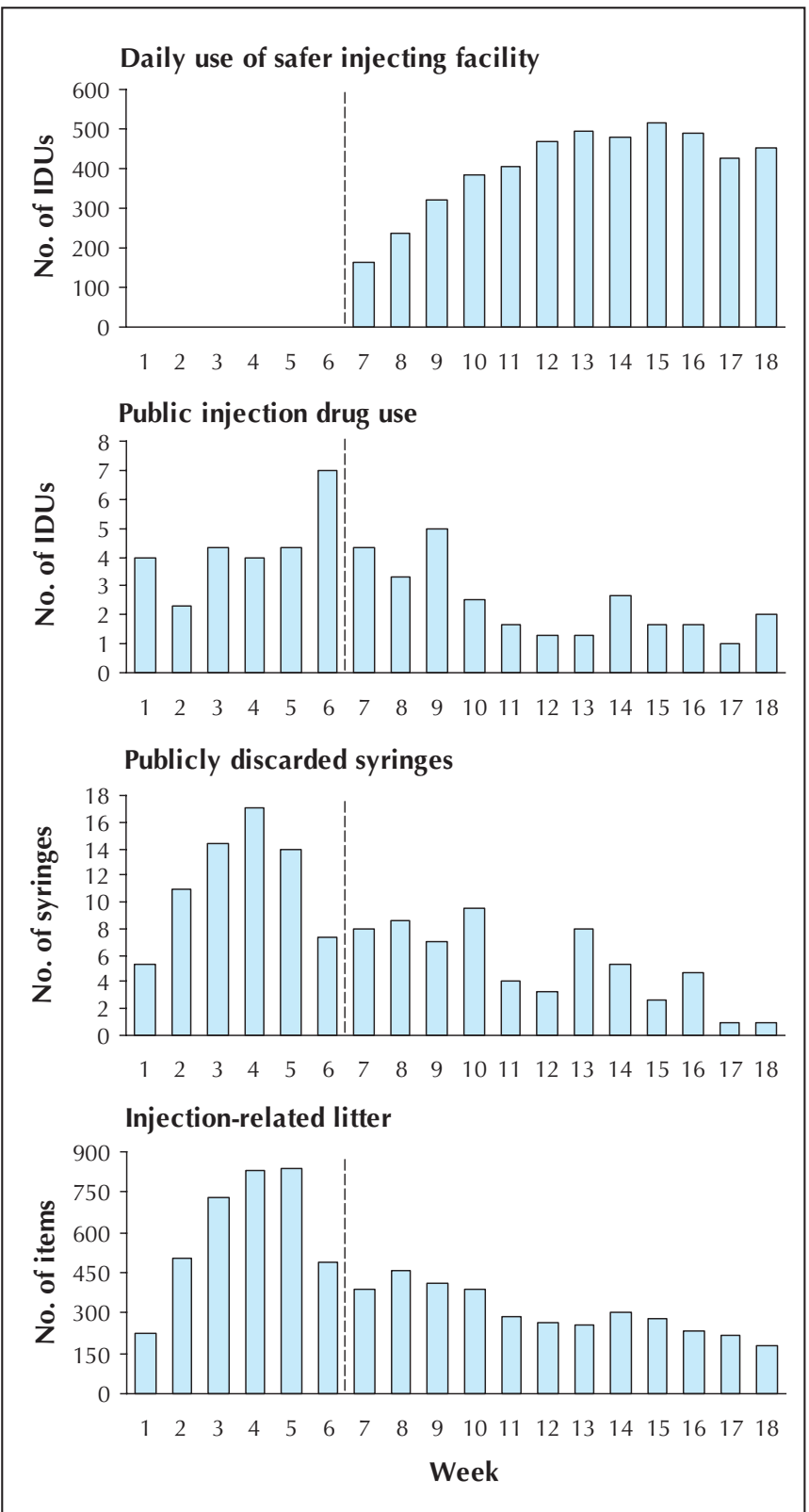

Fig. 1: Mean daily numbers of injection drug users (IDUs) who visited Vancouver's safer injecting facility, IDUs who injected in public, publicly discarded syringes and injection-related litter counted during the 6 weeks before and the 12 weeks after the facility opened. Dotted line represents opening of facility. 


\section{Results}

The operating hours of Vancouver's safer injecting facility were $10 \mathrm{am}$ to $4 \mathrm{am}$ every day. The mean number of visits to the facility in the first week of operation was 184; this number increased to 504 visits 2 months later (Fig. 1).

When we compared data for the periods before and after the opening of the facility, we found statistically significant reductions in the daily mean numbers of IDUs injecting in public (4.3 [interquartile range (IQR) 4.0-4.3] v. 2.4 [IQR $1.5-3.0] ; p=0.022)$, publicly discarded syringes (11.5 [IQR $7.3-14.3$ v. 5.3 [IQR 3.0-8.0]; $p=0.010$ ) and injectionrelated litter (601.7 [IQR 490.0-830.3] v. 305.3 [IQR 246.3387.0]; $p=0.014$ ) (Fig. 1). When we tested for correlations between daily counts of facility usage and daily counts of the 3 public order measures, we found that the correlations were statistically significant $(p<0.001)$ in each case (public injection drug use, $r=-0.48$; publicly discarded syringes, $r=$ -0.56 ; and injection-related litter, $r=-0.62$ ). The daily mean number of suspected drug dealers was 45.2 in the period before and 40.7 in the period after the opening of the facility; the difference was not statistically significant $(p=0.34)$.

In the Poisson log-linear regression model in which the number of IDUs injecting in public per day was the dependent variable, the period after the opening of the safer injecting facility was associated with a statistically significant reduction in the count $(\beta$ coefficient $=-0.59 ; p<0.001$ ), whereas daily rainfall $(\beta$ coefficient $=-0.008 ; p=0.42)$ and police presence $(\beta$ coefficient $=0.004 ; p=0.91)$ were not. In the model considering the number of publicly discarded syringes observed per day, all 3 variables were independently associated with a reduction in the number: period after opening of facility, $\beta$ coefficient $=-0.76(p<0.001)$; daily rainfall, $\beta$ coefficient $=-0.02(p=0.025)$; and police presence, $\beta$ coefficient $=0.05(p=0.040)$. Similarly, in the third model the 3 variables were independently associated with a reduction in the count of injection-related litter observed per day: period after opening of facility, $\beta$ coefficient $=$ $-0.66(p<0.001)$; daily rainfall, $\beta$ coefficient $=-0.006(p<$ $0.001)$; and police presence, $\beta$ coefficient $=0.04(p<0.001)$. After adjustment for rainfall and police presence, the period after the opening of the facility remained associated with a reduction in public injection drug use ( $\beta$ coefficient $=-0.61 ; p<0.001)$, publicly discarded syringes $(\beta$ coefficient $=-0.72 ; p<0.001)$ and injection-related litter $(\beta$ coefficient $=-0.72 ; p<0.001)$.

Using the parameter estimates from the unadjusted regression model, we calculated the predicted mean daily level of each public order measure in the periods before and after the opening of the safer injecting facility (Table 1). The predicted mean daily number of IDUs injecting drugs in public 4.3 (95\% CI 3.5-5.4) before the facility opened and 2.4 (95\% CI 1.9-3.0) after it opened. The corresponding values were 11.5 (95\% CI 10.0-13.2) and 5.4 (95\% CI 4.7-6.3) for the predicted daily mean number of publicly discarded syringes and 601 (95\% CI 590-613) and
310 (95\% CI 305-317) for the predicted daily mean count of injection-related litter.

When we examined the number of syringes discarded in the neighbourhood's 6 outdoor safe disposal boxes, the mean number per box per week was significantly higher before than after the safer injecting facility opened (30.9 v. 9.4; $p<0.001)$.

\section{Interpretation}

We found significant reductions in public injection drug use, publicly discarded syringes and injection-related litter after the opening of the medically supervised safer injecting facility in Vancouver. These reductions were independent of law enforcement activities and changes in rainfall patterns.

Our findings are consistent with anecdotal reports of improved public order following the establishment of safer injecting facilities ${ }^{12,15}$ and are not surprising given that a commonly reported reason for public drug use is the lack of an alternative place to inject and that IDUs who go to safer injecting facilities are often homeless or marginally housed. ${ }^{26}$ Our findings are also highly plausible since more than 500 IDUs visited the facility daily after it opened, and several feasibility studies have suggested that IDUs who inject in public would be the most likely to use safer injecting facilities. ${ }^{13,27}$ Our observations suggest that the establishment of the safer injecting facility has resulted in measurable improvements in public order, which in turn may improve the liveability of communities and benefit tourism while reducing community concerns stemming from public drug use and discarded syringes. ${ }^{7-10}$ It is also noteworthy that we did not observe an increase in the number of drug dealers in the vicinity of the facility, which indicates that the facility's opening did not have a negative impact on drug dealing in the area. Although further study of these issues is necessary, the safer injecting facility may also offer public health benefits, since public injection drug use has been associated with an array of health-related harms. ${ }^{11,12,18}$

Our study has limitations. Although we attempted to reduce the effect of seasonality by limiting the duration of the study, a seasonal fluctuation in drug use patterns may have

Table 1: Predicted daily mean measures of public order problems during the 6 weeks before and the 12 weeks after the opening of Vancouver's safer injecting facility*

\begin{tabular}{lcc}
\hline & \multicolumn{2}{c}{$\begin{array}{c}\text { Predicted daily mean no. } \\
\text { (and 95\% Cl) }\end{array}$} \\
\cline { 2 - 3 } Measure & $\begin{array}{c}\text { Before the facility } \\
\text { opened }\end{array}$ & $\begin{array}{c}\text { After the facility } \\
\text { opened }\end{array}$ \\
\hline IDUs injecting in public & $4.3(3.5-5.4)$ & $2.4(1.9-3.0)$ \\
Publicly discarded syringes & $11.5(10.0-13.2)$ & $5.4(4.7-6.3)$ \\
Injection-related litter & $601(590-613)$ & $310(305-317)$ \\
\hline
\end{tabular}

Note: $\mathrm{Cl}=$ confidence interval.

* Parameter estimates from the unadjusted Poisson log-linear regression models were used to calculate the predicted means (see Methods for details). 
affected our findings. However, our estimates did not change significantly after adjustment for daily rainfall statistics, and seasonal reductions in public drug use have not been previously observed in Vancouver. ${ }^{23,28}$ The uncontrolled nature of our study also raises the potential for an observer bias. This bias, if it existed, is an unlikely explanation sinced our findings are consistent with anecdotal reports from police and other agencies in the neighbourhood that have reported reduced public injection drug use in the wake of the safer injecting facility's opening, ${ }^{29-31}$ and police have reportedly been helping IDUs find the facility. Furthermore, our findings were consistent with the city's compiled data regarding discarded syringes in the outdoor safe disposal boxes.

In summary, we documented significant reductions in the number of IDUs injecting in public, publicly discarded syringes and injection-related litter after the opening of the medically supervised safer injecting facility. These reductions appeared to be independent of several potential confounders, and our findings were supported by external data sources. Although the overall health impacts of the facility will take several years to evaluate, the findings from this study should be valuable to other cities that are contemplating similar evaluations and should have substantial relevance to many urban areas where public injection drug use has been associated with substantial public health risks ${ }^{11,32,33}$ and adverse community impacts. ${ }^{11-13}$

\section{This article has been peer reviewed.}

From the British Columbia Centre for Excellence in HIV/AIDS, St. Paul's Hospital, Vancouver, BC (all authors); the Department of Health Care and Epidemiology (Wood) and the Department of Medicine (Montaner, Tyndall), University of British Columbia, Vancouver, BC; the Canadian HIV/AIDS Legal Network, Montréal, Que. (Kerr); and Vancouver Coastal Health (Marsh), Vancouver, BC

Competing interests: None declared.

Contributors: Evan Wood, Mark Tyndall and Will Small were responsible for the study design. Will Small collected the field survey data. Kathy Li performed all statistical analyses. Evan Wood wrote the first draft of the manuscript and compiled the coauthors suggestions. All of the authors contributed to the conceptualization of the study and the various drafts of the manuscript and approved the final version.

Acknowledgements: We thank the staff of the Insite safer injecting facility and Vancouver Coastal Health (Chris Buchner, Heather Hay). We also thank Bonnie Devlin, Evelyn King, Aaron Eddie, Peter Vann, Dave Isham, Steve Gaspar, Car Bognar, Martin Schechter, Suzy Coulter and Steve Kain for their administrative assistance and suggestions.

This study was made possible by a financial contribution from Health Canada, although the views expressed herein do not represent the official policies of Health Canada.

\section{References}

1. Strathdee SA, Galai N, Safaiean M, Celentano DD, Vlahov D, Johnson L, et al. Sex differences in risk factors for HIV seroconversion among injection drug users: a 10-year perspective. Arch Intern Med 2001;161(10):1281-8.

2. Des Jarlais DCP, Friedman SRP, Hagan HMPH. Maintaining low HIV seroprevalence among injecting drug users. 7AMA 1996;275(8):597-8.

3. Tyndall MW, Currie S, Spittal P, Li K, Wood E, O'Shaughnessy MV, et al. Intensive injection cocaine use as the primary risk factor in the Vancouver HIV-1 epidemic. AIDS 2003;17(6):887-93.

4. Garfield J, Drucker E. Fatal overdose trends in major US cities: 1990-97. Addict Res Theory 2001;9(5):425-36.

5. Wood E, Tyndall MW, Spittal PM, Li K, Kerr T, Hogg RS, et al. Unsafe injection practices in a cohort of injection drug users in Vancouver: Could safer injecting rooms help? CMA7 2001;165(4):405-10.
6. Coffin PO, Galea S, Ahern J, Leon AC, Vlahov D, Tardiff K. Opiates, cocaine, and alcohol combinations in accidental drug overdose deaths in New York City, 1990-98. Addiction 2003;98(6):739-47.

7. Doherty MC, Garfein RS, Vlahov D, Junge B, Rathouz PJ, Galai N, et al Discarded needles do not increase soon after the opening of a needle exchange program. Am 7 Epidemiol 1997;145(8):730-7.

8. Doherty MC, Junge B, Rathouz P, Garfein RS, Riley E, Vlahov D. The effect of a needle exchange program on numbers of discarded needles: a 2-year follow-up. Am 7 Public Health 2000;90(6):936-9.

9. Broadhead RS, van Hulst Y, Heckathorn DD. The impact of a needle exchange's closure. Public Health Rep 1999;114(5):439-47.

10. Oliver KJ, Friedman SR, Maynard H, Magnuson L, Des Jarlais DC. Impact of a needle exchange program on potentially infectious syringes in public places. 7 Acquir Immune Defic Syndr 1992;5(5):534-5.

11. Dovey K, Fitzgerald J, Choi Y. Safety becomes danger: dilemmas of drug-use in public space. Health Place 2001;7(4):319-31.

12. Broadhead RS, Kerr TH, Altice FL. Safer injection facilities in North America: their place in public policy and health initiatives. 7 Drug Issues 2002;32(1):329-55.

13. Wood E, Kerr T, Spittal PM, Li K, Small W, Tyndall MW, et al. The potential public health and community impacts of safer injecting facilities: evidence from a cohort of injection drug users. 7 Acquir Immune Defic Syndr 2003;32(1):2-8

14. Kimber J, Dolan K, van Beek I, Hedrich D, Zurhold H. Drug consumption facilities: an update since 2000. Drug Alcohol Rev 2003;22(2):227-33.

15. Dolan K, Kimber J, Fry C, Fitzgerald J, McDonald D, Frautmann F. Drug consumption facilities in Europe and the establishment of supervised injecting centres in Australia. Drug Alcobol Rev 2000;19:337-46.

16. De Jong $W$, Wever $U$. The professional acceptance of drug use: a closer look at drug consumption rooms in the Netherlands, Germany, and Switzerland. Int 7 Drug Policy 1999; 10:99-108.

17. Final report of the evaluation of the Sydney Medically Supervised Injecting Centre. Kings Cross (Australia): Sydney Medically Supervised Injecting Centre; Available: www.sydneymsic.com/Bginfo.htm [click on link to PDF of report] (accessed 2004 June 17).

18. Wood E, Kerr T, Montaner JS, Strathdee SA, Wodak A, Hankins CA, et al. Rationale for evaluating North America's first medically supervised safer injecting facility. Lancet Infect Dis 2004;4:301-6.

19. Strathdee SA, Patrick DM, Currie SL, Cornelisse PG, Rekart ML, Montaner JS, et al. Needle exchange is not enough: lessons from the Vancouver injecting drug use study. AIDS 1997;11(8):F59-65.

20. Wood E, Schechter MT, Tyndall MW, Montaner JS, O'Shaughnessy MV Hogg RS. Antiretroviral medication use among injection drug users: two potential futures. AIDS 2000;14(9):1229-35.

21. Wood E, Spittal PM, Small W, Kerr T, Li K, Hogg RS, et al. Displacement of Canada's largest public illicit drug market in response to a police crackdown. CMA7 2004;170(10):1551-6.

22. Kerr T, Small W, Peeace W, Pierre A, Wood E. Harm reduction by a 'userrun' organization: a case study of the Vancouver area network of drug users. Int 7 Drug Policy In press.

23. Spittal P, Small W, Laliberte N, Johnson C, Wood E. How otherwise well meaning exchange agents can contribute to limited sterile syringe availability in Vancouver, Canada. Int 7 Drug Policy 2003;15(1):36-45.

24. Wood E, Kerr T, Small W, Jones J, Schechter MT, Tyndall MW. The impact of police presence on access to needle exchange programs. 7 Acquir Immune Defic Syndr 2003;34(1):116-8.

25. Canadian climate data report. Ottawa: Health Canada. Available: www.climate weatheroffice.ec.gc.ca/climateData/hourlydata_e.html (accessed 2004 June 17).

26. Fry C, Fox S, Rumbold G. Establishing safe injecting rooms in Australia: attitudes of injecting drug users. Aust N Z 7 Public Health 1999;23(5):501-4.

27. Kerr T, Wood E, Small D, Palepu A, Tyndall M. Potential use of safer injecting facilities among injection drug users in Vancouver's Downtown Eastside. CMA7 2003;169(8):759-63.

28. Wood E, Kerr T, Spittal PM, Small W, Tyndall MW, O'Shaughnessy MV et al. An external evaluation of a peer-run "unsanctioned" syringe exchange program. $\mathcal{F}$ Urban Health 2003;80(3):455-64.

29. Safe injection site no problem, say police. Vancouver: CBC News British Columbia; 2004 Mar 3. Available: vancouver.cbc.ca/regional/servlet/View?filename =bc_vpd_sis20040303 (accessed 2004 June 17)

30. Street injections down, police say. Vancouver Sun 2003 Nov 18; Sect B:6.

31. Fewer addicts shooting up on streets since injection site opened: police. Nanaimo Daily News 2003 Nov 18; Sect A:5.

32. Klee H, Morris J. Factors that characterize street injectors. Addiction 1995;90 (6):837-41.

33. Darke S, Kaye S, Ross J. Geographical injecting locations among injecting drug users in Sydney, Australia. Addiction 2001;96(2):241-6.

Correspondence to: Dr. Evan Wood, Division of Epidemiology and Population Health, BC Centre for Excellence in HIV/AIDS, 608-1081 Burrard St., Vancouver BC V6Z 1Y6;

fax 604 806-9044; ewood@cfenet.ubc.ca 